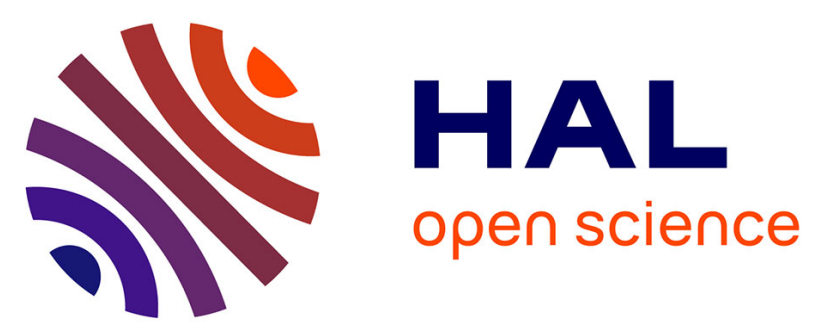

\title{
Detection \& isolation of sensor and actuator additive faults in a 4-mecanum wheeled mobile robot (4-MWMR)
}

Samia Mellah, Guillaume Graton, El Mostafa El Adel, Mustapha Ouladsine, Alain Planchais

\section{- To cite this version:}

Samia Mellah, Guillaume Graton, El Mostafa El Adel, Mustapha Ouladsine, Alain Planchais. Detection \& isolation of sensor and actuator additive faults in a 4-mecanum wheeled mobile robot (4MWMR). ICCAD 2019: IEEE-International Conference on Control, Automation and Diagnosis -, Jul 2019, Grenoble, France. hal-02381863

\section{HAL Id: hal-02381863 https://hal.science/hal-02381863}

Submitted on 26 Nov 2019

HAL is a multi-disciplinary open access archive for the deposit and dissemination of scientific research documents, whether they are published or not. The documents may come from teaching and research institutions in France or abroad, or from public or private research centers.
L'archive ouverte pluridisciplinaire HAL, est destinée au dépôt et à la diffusion de documents scientifiques de niveau recherche, publiés ou non, émanant des établissements d'enseignement et de recherche français ou étrangers, des laboratoires publics ou privés. 


\title{
Detection \& isolation of sensor and actuator additive faults in a 4-mecanum wheeled mobile robot (4-MWMR)
}

\author{
Samia MELLAH ${ }^{1}$, Guillaume GRATON ${ }^{1,2}$, El Mostafa EL ADEL ${ }^{1}$, Mustapha OULADSINE ${ }^{1}$ and \\ Alain PLANCHAIS ${ }^{3}$
}

\begin{abstract}
In this paper, the fault detection and isolation problem regarding actuation and sensing of a 4-mecanum wheeled mobile robot (4-MWMR) is studied. The challenge with respect to the current state of the art lies in detecting and distinguishing wheel sensor from wheel actuator additive faults for this kind of robots. An approach based on generating residuals is proposed. Sensor faults isolation is based on simply analyzing residual signatures which are different under each sensor fault. Due to omni-move properties, actuator faults are, however, more difficult to be isolated. More residual characteristics must be taken into consideration to achieve the isolation.

Keywords: 4-mecanum mobile robot, kinematic and dynamic model, residual generation, residual signature, extended Kalman observer, sensor and actuator additive faults.
\end{abstract}

\section{INTRODUCTION}

Nowadays, autonomous wheeled mobile robots (whether they are unicycle, car-like, or omni-move robots), are widely solicited in industry where they play an important role. Their strong point lies into their capability of replacing humans to accomplish easily repetitive, difficult, or risky activities.

Unfortunately, although their capacity of working for several hours without stooping, the appearance of faults in wheeled mobile robots (WMRs), like in all embedded systems, is inevitable. This can be due to either components wear, or to the WMRs environment which can present unanticipated situations. Such faults can appear in the battery level, in the positioning system, sensor, or actuator level, ...

Any non-permitted deviation of at least one property of a system from the acceptable condition can represent a fault. A fault can be additive or multiplicative and it can lead to the entire system failure.

In order to be efficient and largely smart, WMRs should not only be able to localize their selves, to sense their surroundings (perception), to generate a path to their destination (path planning) and to execute it (navigation) in an efficient manner, but they should also be able to do selfdiagnosis (i.e, to detect and isolate internal faults without human intervention).

*Research leading to these results has received funding from the EU ECSEL Joint Undertaking under grant agreement n 737459 (project Productive4.0) and from the partners national funding authorities DGE.

${ }^{1}$ S. Mellah, G. Graton, E. M. El Adel and M. Ouladsine are with Aix Marseille Univ, Université de Toulon, CNRS, LIS (UMR 7020), Avenue Escadrille Normandie-Niemen, F-13397 Marseille Cedex 20, France samia.mellah@lis-lab.fr

${ }^{2}$ G. Graton is with Ecole Centrale Marseille, Technopôle de ChâteauGombert, 38 rue Frédéric Joliot-Curie, F-13451 Marseille Cedex 13, France

${ }^{3}$ A. Planchais is with ST Microelectronics Rousset, 190 avenue Celestin Coq, ZI - Rousset-Peynier, F-13106 Rousset, France
Fault detection \& isolation (FDI) methods can be classified into two main categories, namely history or data-based methods and model-based methods. Data-based methods require a collection and manipulation of a large quantity of historic data, while model-based methods need mathematical models developed from the fundamental understanding of systems physics. According to [6], several model-based diagnosis techniques have been successfully implemented on real systems. This motivates us to be interested in these approaches.

Concerning WMRs FDI, several efforts have been concentrated on detecting \& isolating either sensor or actuator faults using model-based approaches. A state of the art about WMRs FDI and fault tolerant control is given in [2]. In [1], some model-based techniques are presented for WMRs FDI.

Authors in [3] propose to use Kalman filter identification technique to detect and isolate car-like WMRs sensor faults. For the same type of WMRs, a structural analysis-based approach is proposed for actuator FDI in [4].

This work is focused on omnidirectional mobile robots. Thanks to their maneuvering capabilities with respect to nonholonomic mobile robots (i.e, their ability of moving in any direction without any reorientation), they have received more attention in mobile robot applications, especially in dynamic environments such as transportation, military, space, health-care, ...

This work is sponsored by PRODUCTIVE4.0, with an application in STMicroelectronics company. In order to robotize the semiconductors production fab, STMicroelectronics company plans to use omnidirectional mobile robots to transport batches from one equipment to another.

The purpose of our work is to insure the reliability of the robots, by detecting as soon as possible any malfunction that could lead to an unpredictable robot failure. Detect and isolate a fault allows to plan a corrective maintenance without having to stop immediately the robot during production. The goal is to avoid serious economic consequences.

Regarding omnidirectional mobile robots, many works have been focused on modeling and developing control laws to improve more and more their autonomy and intelligence (see [8], [9], [11], [12] and references therein).

In [10], a three omnidirectional mobile robot FDI is considered using a bank of extended Kalman filters (EKF) and a bank of particle filters. A comparative study between the two approaches shows that the EKF is more efficient.

In [11], a 4-mecanum wheeled mobile robot fault tolerant control is proposed under actuator faults.

For the best of our knowledge, no work has been published 
in the literature regarding 4-mecanum wheeled mobile robots (4-MWMRs) FDI problem.

In this paper, sensor and actuator additive faults are studied. An observer-based technique is proposed to detect and distinguish wheel sensor from wheel actuator additive faults in a 4-MWMRs. The method is based on estimating, in parallel to the system, the robot state space vectorat each instant $k$ using an extended Kalman observer (EKO). Then, based on comparing the system and the observer outputs, residuals are generated and analyzed.

The challenge of this work lies on the complete isolation of wheel sensor and wheel actuator small additive faults as early as possible. These faults can represent components wear.

The paper is organized as follows: in section II, a 4MWMR kinematic and dynamic models are presented, and the robot omni motions principle is explained. In section III, residual generation and fault isolation principle are detailed. Simulation results are done in section IV for illustration, followed by a conclusion and perspectives in the last section.

\section{4-MWMR MATHEMATICAL MODEL}

Fig. 1 shows a 4-MWMR geometry. To consider the kinematic model, it is assumed that the robot is placed on a plane surface where $(O, \vec{x}, \vec{y})$ is the inertial reference frame and $\left(G, \overrightarrow{x_{R}}, \overrightarrow{y_{R}}\right)$ is a local coordinate frame fixed on the robot at its center of mass, which coincides with its geometric center $G$.

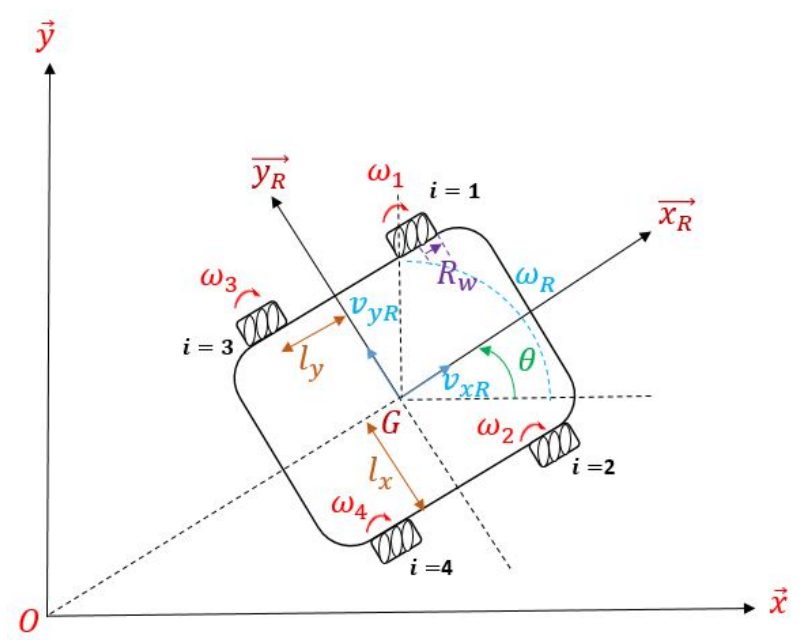

Fig. 1: 4-mecanum wheeled robot geometry.

Following assumptions are made:

- The WMR is equipped with four optical encoders $E_{i}$, $(i=1, \ldots 4)$, they return in real time the 4-wheels angular velocities $\omega_{i},(i=1, \ldots 4)$, and with a gyroscope to provide the robot orientation angle $\theta$ (see Fig. 1).

- $x$ and $y$ are given by a positioning system $(e ; g$, WIFI, GPS, ...).

\section{A. Nomenclature}

The following notations will be used throughout this article (see Fig. 1) and Table I.

\begin{tabular}{|l|l|}
\hline \multicolumn{1}{|c|}{ Variable } & \multicolumn{1}{c|}{ Description \& unit } \\
\hline$x, y$ & Robot position along X-axes and $\mathrm{y}$-axes $(\mathrm{m})$. \\
$l_{x}$ & Robot orientation angle $(\mathrm{rad})$. \\
$l_{y}$ & Half distance between front wheels $(\mathrm{m})$. \\
$l=l_{x}+l_{y}$ & Half distance between front and rear wheels $(\mathrm{m})$. \\
$R_{w}$ & Wheel radius (m). \\
$\omega_{i}=\dot{\theta}_{i}$ & Wheel angular velocities $(\mathrm{rad} / \mathrm{s})$. \\
$\dot{\omega}_{i}=\ddot{\theta}_{i}$ & Wheel angular accelerations $\left(\mathrm{rad} / \mathrm{s}^{2}\right)$. \\
$v_{x}=\dot{x}, v_{y}=\dot{y}$ & Robot linear velocities $(\mathrm{m} / \mathrm{s})$. \\
$\omega_{R}=\dot{\theta}$ & Robot angular velocity $(\mathrm{rad} / \mathrm{s})$. \\
$I_{z}$ & Moment of inertia of the platform $\left(\mathrm{kg} . \mathrm{m}^{2}\right)$. \\
$I_{w}$ & Moment of inertia of the wheels $\left(\mathrm{kg} \cdot \mathrm{m}^{2}\right)$. \\
$m$ & Robot overall mass (m). \\
$\tau_{i}$ & Applied torque to wheel $i(\mathrm{~N} . \mathrm{m})$. \\
\hline
\end{tabular}

TABLE I: Nomenclature

\section{B. Kinematic model}

1) Inverse kinematic model: The inverse kinematic model is given by: (see [9] for more details)

$$
\left[\begin{array}{l}
\omega_{1} \\
\omega_{2} \\
\omega_{3} \\
\omega_{4}
\end{array}\right]=J\left[\begin{array}{l}
v_{x R} \\
v_{y R} \\
\omega_{R}
\end{array}\right], J=\frac{1}{R_{w}}\left[\begin{array}{ccc}
1 & -1 & -l \\
1 & 1 & l \\
1 & 1 & -l \\
1 & -1 & l
\end{array}\right]
$$

2) Forward kinematic model: The forward kinematic model is given by: (see [9] for more details)

$$
\left[\begin{array}{c}
v_{x R} \\
v_{y R} \\
\omega_{R}
\end{array}\right]=J^{+}\left[\begin{array}{l}
\omega_{1} \\
\omega_{2} \\
\omega_{3} \\
\omega_{4}
\end{array}\right]
$$

with $J^{+}$the pseudo-inverse of the matrix $J$ as follows:

$$
J^{+}=\left(J^{T} J\right)^{-1} J^{T}=\frac{R_{w}}{4}\left[\begin{array}{cccc}
1 & 1 & 1 & 1 \\
-1 & 1 & 1 & -1 \\
\frac{-1}{l} & \frac{1}{l} & \frac{-1}{l} & \frac{1}{l}
\end{array}\right]
$$

3) Inertial frame model expression: By denoting $c=\cos \theta$ and $d=\sin \theta$, the robot velocities are expressed in the inertial frame $(O, \vec{x}, \vec{y})$ using the following transformation matrix $T$ (see [11])

$$
\left[\begin{array}{c}
\dot{x} \\
\dot{y} \\
\dot{\theta}
\end{array}\right]=T\left[\begin{array}{c}
v_{x R} \\
v_{y R} \\
\omega
\end{array}\right], T=\left[\begin{array}{ccc}
c & -d & 0 \\
d & c & 0 \\
0 & 0 & 1
\end{array}\right]
$$

Using this transformation (4), the robot forward kinematic model (2) is expressed in $(O, \vec{x}, \vec{y})$ as follows:

$$
\left[\begin{array}{l}
\dot{x} \\
\dot{y} \\
\dot{\theta}
\end{array}\right]=E\left[\begin{array}{l}
\omega_{1} \\
\omega_{2} \\
\omega_{3} \\
\omega_{4}
\end{array}\right]
$$

where

$$
E=T J^{+}=\frac{R_{w}}{4}\left[\begin{array}{cccc}
c+d & c-d & c-d & c+d \\
d-c & c+d & c+d & d-c \\
\frac{-1}{l} & \frac{1}{l} & \frac{-1}{l} & \frac{1}{l}
\end{array}\right]
$$




\section{Dynamic model}

The Lagrangian equation is given by the difference between $K$, the robot kinetic energy and $P$, its potential energy. Since the robot moves on a planar surface, $P=0 . K$ can be calculated as follows: [11]

$$
K=\frac{1}{2} m\left(\dot{x}^{2}+\dot{y}^{2}\right)+\frac{1}{2} I_{z} \omega_{R}^{2}+\frac{1}{2} I_{w}\left(\omega_{1}+\omega_{2}+\omega_{3}+\omega_{4}\right)
$$

where, $\dot{x}, \dot{y}$, and $\omega_{R}=\dot{\theta}$ are given by (5) and (6). Neglecting the loss of energy owing to the wheel viscous friction and invoking the Euler Lagrange equation, the robot dynamic is given by: (see [11] for more details)

$$
M \dot{\omega}=\tau
$$

$\dot{\omega}=\left[\dot{\omega}_{1}, \dot{\omega}_{2}, \dot{\omega}_{3}, \dot{\omega}_{4}\right]^{T}, \tau=\left[\tau_{1}, \tau_{2}, \tau_{3}, \tau_{4}\right]^{T}$, and $M$ is a $(4 \times 4)$ matrix given by:

$$
M=\left[\begin{array}{cccc}
a+b+I_{w} & -b & b & a-b \\
-b & a+b+I_{w} & a-b & b \\
b & a-b & a+b+I_{w} & -b \\
a-b & b & -b & a+b+I_{w}
\end{array}\right]
$$

with $a=\frac{m R_{w}^{2}}{8}$ and $b=\frac{I_{z} R_{w}^{2}}{16 l}$.

Since $M$ is a invertible matrix, the wheel angular accelerations $\dot{\omega}_{i}, i=1, \ldots, 4$ can be expressed with respect to the applied torques as follows:

$$
\dot{\omega}=S \tau
$$

with $S=M^{-1}$.

\section{Augmented state space representation}

Combining the forward kinematic and dynamic models ((5) and (10)), the robot global model can be obtained in the global inertial reference frame:

$$
\left[\begin{array}{c}
\dot{x} \\
\dot{y} \\
\dot{\theta} \\
\dot{\omega}_{1} \\
\dot{\omega}_{2} \\
\dot{\omega}_{3} \\
\dot{\omega}_{4}
\end{array}\right]=\left[\begin{array}{c}
f_{1}\left(\theta, \omega_{i}\right) \\
f_{2}\left(\theta, \omega_{i}\right) \\
f_{3}\left(\theta, \omega_{i}\right) \\
0 \\
0 \\
0 \\
0
\end{array}\right]+\left[\begin{array}{cccc}
0 & 0 & 0 & 0 \\
0 & 0 & 0 & 0 \\
0 & 0 & 0 & 0 \\
s_{11} & s_{12} & s_{13} & s_{14} \\
s_{21} & s_{22} & s_{23} & s_{24} \\
s_{31} & s_{32} & s_{33} & s_{34} \\
s_{41} & s_{42} & s_{43} & s_{44}
\end{array}\right]\left[\begin{array}{c}
\tau_{1} \\
\tau_{2} \\
\tau_{3} \\
\tau_{4}
\end{array}\right]
$$

where $f_{j}\left(\theta, \omega_{i}\right),(j=1, \ldots, 3)$ and $(i=1, \ldots 4)$ are given by (5) and (6).

Finally, the global model in the presence of model uncertainties and measurements noise, is given in state space representation (SSR):

$$
\left\{\begin{array}{l}
\dot{X}=F(X, t)+D u+\eta(t) \\
Y=C X+\gamma(t)
\end{array}\right.
$$

where $X$ is the state vector, $u$ the control input vector (torques), $Y$ the measurements vector (which is equal to $X$ according to assumptions given in the beginning of this section II. Thus, $C$ is a $(n \times n)$ identity matrix $(n=7$ is the system dimension), $D=\left(\begin{array}{c}0_{3 \times 4} \\ S_{4 \times 4}\end{array}\right)$, and $\eta, \gamma$ are respectively model uncertainties and measurement noise.
They are supposed to be white noises with known covariance matrices, respectively $Q$, and $R$. Also, $Q$ is assumed having no cross-correlation with $R$.

Since measurements are not given in continuous time, the SSR is discretized using Euler's method, respecting sampling time $T_{s}$ :

$$
\left\{\begin{aligned}
X(k+1) & =X(k)+T_{s}(F(X, k)+D u(k)+\eta(k)) \\
Y(k) & =C X(k)+\gamma(k)
\end{aligned}\right.
$$

\section{E. Omni-motion principle}

Table II below resumes the different obtained movements by controlling the omnidirectionnal wheels with different velocity combinations $\left(C_{1}\right.$ and $C_{2}$, two constants given respectively in $\mathrm{m} / \mathrm{s}$ and $\mathrm{rad} / \mathrm{s}$ ). See [9] for more details.

\begin{tabular}{|l|l|l|l|l|l|l|l|}
\hline Motion & $v_{x R}$ & $v_{y R}$ & $\omega_{R}$ & $\omega_{1}$ & $\omega_{2}$ & $\omega_{3}$ & $\omega_{4}$ \\
\hline Forward & $\mathrm{C}_{1}$ & 0 & 0 & $\mathrm{C}_{2}$ & $\mathrm{C}_{2}$ & $\mathrm{C}_{2}$ & $\mathrm{C}_{2}$ \\
\hline Backward & $-\mathrm{C}_{1}$ & 0 & 0 & $-\mathrm{C}_{2}$ & $-\mathrm{C}_{2}$ & $-\mathrm{C}_{2}$ & $-\mathrm{C}_{2}$ \\
\hline Left & 0 & $\mathrm{C}_{1}$ & 0 & $-\mathrm{C}_{2}$ & $\mathrm{C}_{2}$ & $\mathrm{C}_{2}$ & $-\mathrm{C}_{2}$ \\
\hline Right & 0 & $-\mathrm{C}_{1}$ & 0 & $\mathrm{C}_{2}$ & $-\mathrm{C}_{2}$ & $-\mathrm{C}_{2}$ & $\mathrm{C}_{2}$ \\
\hline $\begin{array}{l}\text { Left diagonal } \\
\text { forward }\end{array}$ & $\mathrm{C}_{1}$ & $\mathrm{C}_{1}$ & 0 & 0 & $2 \mathrm{C}_{2}$ & $2 \mathrm{C}_{2}$ & 0 \\
\hline $\begin{array}{l}\text { Left diagonal } \\
\text { backward }\end{array}$ & $-\mathrm{C}_{1}$ & $\mathrm{C}_{1}$ & 0 & $-2 \mathrm{C}_{2}$ & 0 & 0 & $-2 \mathrm{C}_{2}$ \\
\hline $\begin{array}{l}\text { Right diagonal } \\
\text { forward }\end{array}$ & $\mathrm{C}_{1}$ & $-\mathrm{C}_{1}$ & 0 & $2 \mathrm{C}_{2}$ & 0 & 0 & $2 \mathrm{C}_{2}$ \\
\hline $\begin{array}{l}\text { Right diagonal } \\
\text { backward }\end{array}$ & $-\mathrm{C}_{1}$ & $-\mathrm{C}_{1}$ & 0 & 0 & $-2 \mathrm{C}_{2}$ & $-2 \mathrm{C}_{2}$ & 0 \\
\hline
\end{tabular}

TABLE II: Motions of four omnidirectional mobile robot according to [9].

\section{RESIDUAL-BASED FAULT DETECTION AND ISOLATION}

Generally speaking, model-based FDI methods are based on residual generation. Residuals can be defined as a difference between a system measured and estimated outputs. Measurements are provided by sensors while estimations are obtained using observers.

In normal operating conditions (i.e, not faulty mode), the system and the mathematical model have similar outputs, residuals are close to zero. In the presence of a fault, residuals being sensitive to the concerned fault get away from zero.

\section{A. Studied faults}

The objective is to detect and isolate wheel sensor and actuator additive faults of a 4-MWMR, namely:

Encoder faults $E_{i},\left(i=(1, \ldots, 4)\right.$, and gyroscope fault $G_{r}$. Actuator faults $A_{i},(i=(1, \ldots, 4)$.

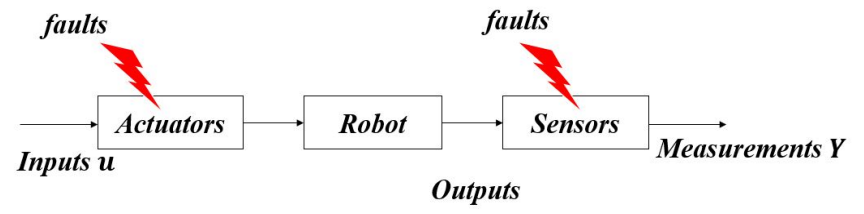

Fig. 2: Studied faults.

In the presence of actuator and sensor additive faults, the system (13) is given by: 


$$
\left\{\begin{aligned}
X(k+1)= & X(k)+T_{s}(F(X, k)+D u(k) \\
& \left.+E_{a} f_{a}(k)+\eta(k)\right) \\
Y(k)= & C X(k)+E_{s} f_{s}(k)+\gamma(k)
\end{aligned}\right.
$$

where $E_{a}$ and $E_{s}$ are respectively actuator and sensor fault matrices with appropriate dimensions, and $f_{a}(k), f_{s}(k)$ are actuator and sensor additive fault vectors.

\section{B. Residual generation}

Extended Kalman observer (EKO) is used to estimate at each instant $k$ the system state $X(k)$.

$\eta$ and $\gamma$ characteristics given for model and measurement noises in (12) are adequate to use EKO.

Kalman observer is based on two steps: a prediction step and a correction step. To predict the state $X$ at time $k+1$ knowing $k$, EKO is defined for the system (14) as follows:

$$
\left\{\begin{aligned}
\hat{X}(k+1 \mid k) & =\hat{X}(k \mid k)+T_{s}(F(\hat{X}, k)+D u(k)) \\
P(k+1 \mid k) & =A_{l}(k) P(k \mid k) A_{l}^{T}(k)+Q
\end{aligned}\right.
$$

where $P(k+1 \mid k)$ denotes the a priori error covariance matrix associated to $X$ and $A_{l}$ the linearized matrix of function $F$ given by $A_{l}(k)=I+\left.T_{s} \frac{\partial F}{\partial X}\right|_{\hat{X}_{(k \mid k)}}$, where:

$$
\left.\frac{\partial F}{\partial X}\right|_{\hat{X}_{(k \mid k)}}=\left[\begin{array}{ll}
\frac{\partial F_{1}}{\partial x_{1}} & \frac{\partial F_{1}}{\partial x_{2}} \ldots \frac{\partial F_{1}}{\partial x_{7}} \\
\vdots & \\
\frac{\partial F_{7}}{\partial x_{1}} & \frac{\partial F_{7}}{\partial x_{2}} \ldots \frac{\partial F_{7}}{\partial x_{7}}
\end{array}\right]_{\hat{X}_{(k \mid k)}}
$$

and $I$ is an identity matrix with the appropriate dimension. The correction step is given by:

$$
\left\{\begin{aligned}
\hat{X}(k+1 \mid k+1)= & \hat{X}(k+1 \mid k)+K(k+1) \times \\
& {[Y(k+1)-C \hat{X}(k+1 \mid k)] } \\
K(k+1)= & P(k+1 \mid k) C^{T}\left(C P(k+1 \mid k) C^{T}+R\right)^{-1} \\
P(k+1 \mid k+1)= & (I-K(k+1) C) P(k+1 \mid k)
\end{aligned}\right.
$$

with $K(k+1)$ the filter gain matrix and $P(k+1 \mid k+1)$ is the a posteriori error covariance matrix at time $k+1$.

Using the linear approximation $A_{l}$ of the function $F$, the state estimation error $e(k+1)$ is given for the system (14) as follows:

$$
\begin{aligned}
e(k+1)= & X(k+1)-\hat{X}(k+1) \\
\approx & \left(A_{l}-K(k) C\right) e(k)+E_{a} f_{a}(k) \\
& +\eta(k)-K(k) E_{s} f_{s}(k)-K(k) \gamma(k)(18)
\end{aligned}
$$

In Kalman Observer principle, the gain $K(k)$ is calculated to minimize the error variance matrix trace, whose the expression is not in terms of sensor and actuator faults. Hence, when a fault appears, it is detected by the state estimation error (18).

To insure the observer convergence, matrices $Q, R$, and $P(0 \mid 0)$ must be well defined. In practical use, several databased methods are applied to determine $Q$ and $R$ (see $e . g$, [5] and references therein). In simulation, these matrices are defined as a part related to the added model uncertainties and to noise behavior in the measurement vector. $R$ values correspond on the residual variances in the nominal (i.e not faulty) mode, while $Q$ values must respect the compromise between filtering and delay: very small values lead to a considerable delay on the filter response, while great values do not allow a good filtering. Regarding $P(0 \mid 0)$ matrix, it is initialized large enough to compensate estimation errors.

Based on measurements and EKO estimation outputs, five residuals are generated (see Fig. 3).

$$
\left\{\begin{array}{l}
r_{1}=\theta-\hat{\theta} \\
r_{2}=\omega_{1}-\hat{\omega}_{1} \\
r_{3}=\omega_{2}-\hat{\omega}_{2} \\
r_{4}=\omega_{3}-\hat{\omega}_{3} \\
r_{5}=\omega_{4}-\hat{\omega}_{4}
\end{array}\right.
$$

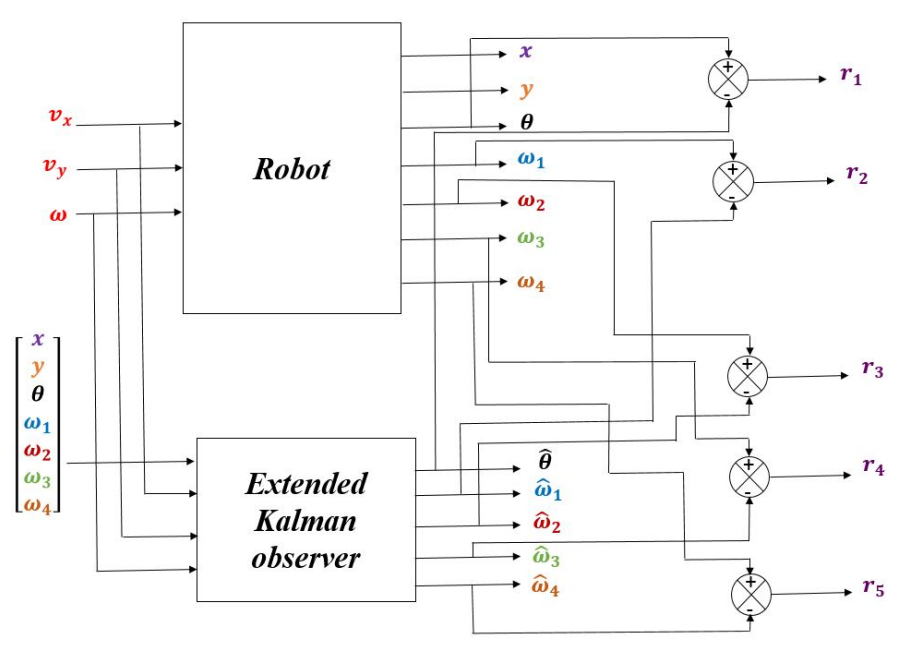

Fig. 3: Residual generation.

\section{Thresholds determination}

Each generated residual $r_{i}$ is compared to 2 threshold values $\pm r_{t h, i}$. Threshold determination is the main difficulty of residual-based FDI methods. Too large thresholds can cause missing alarms, and too small thresholds can cause false alarms.

In the literature, two types of thresholds are distinguished: fixed and adaptive thresholds. Adaptive thresholds are used for inevitable parameter uncertainty, disturbance and noise encountered in practical applications.

In our case, time invariant noises are used in simulations to schematize the system and the measurements noises. Then fixed thresholds are defined using three-sigma method [7]. By defining thresholds with three-sigma method, some false alarms are detected. They must not be taken into consideration. For that, the detection is done when the threshold is exceeded more than $N$ consecutive times. The decision residual $R_{i}$ is turned into 1 when it satisfies the fault handling condition as follows:

$$
R_{i}(k)= \begin{cases}1 & \text { if }\left(r_{i}(j)<-r_{t h, i} \text { or } r_{t h, i}<r_{i}(j)\right), \\ & \forall j \in[k-N+1, k] \\ 0 & \text { otherwise }\end{cases}
$$




\section{Residual signature}

When residuals have different signature under different faults, the isolation is easy. However, when residuals have the same signature under more than one fault, which is the case here, isolation becomes complicated. The residuals (20) signature under the different considered faults are given in Table III.

\begin{tabular}{|c|c|c|c|c|c|c|c|c|c|}
\hline Residuats & $E_{1}$ & $E_{2}$ & $E_{3}$ & $E_{4}$ & $G_{r}$ & $A_{1}$ & $A_{2}$ & $A_{3}$ & $A_{4}$ \\
\hline$R_{1}$ & 0 & 0 & 0 & 0 & 1 & 1 & 1 & 1 & 1 \\
\hline$R_{2}$ & 1 & 0 & 0 & 0 & 0 & 1 & 1 & 1 & 1 \\
\hline$R_{3}$ & 0 & 1 & 0 & 0 & 0 & 1 & 1 & 1 & 1 \\
\hline$R_{4}$ & 0 & 0 & 1 & 0 & 0 & 1 & 1 & 1 & 1 \\
\hline$R_{5}$ & 0 & 0 & 0 & 1 & 0 & 1 & 1 & 1 & 1 \\
\hline
\end{tabular}

TABLE III: Residual signature under wheel sensor and actuator faults.

As it can be noticed, sensor faults are easily isolated. However, actuator faults have the same signature. Relaying only on residual signatures, actuator faults can be detected but not isolated. The isolation is done by taking into consideration other residual properties as explained in the next subsection.

\section{E. Isolation principle}

According to (8), the input vector $\tau$ acts on the system state through the matrix $S=M^{-1}$, which is symmetric. Relying on the matrix $S$ characteristics, each input acts on one wheel more than the others (i.e, the input $\tau_{i}$ acts on the $\omega_{i}$ or on the wheel $i$ more than $\omega_{j},(i=1, \ldots, 4$ and $j \neq$ $i$ ). Hence, a fault on wheel $i$ is detected predominantly by residual $r(i+1)$, followed by residual $r(6-i)$ (see $S$ values and characteristics, section IV). Fault detection percentage by each residual can be calculated at each iteration using the following relation:

$$
r_{i, \text { percentage }}=100 \frac{\max \left|r_{i}(k)\right|}{\sum_{i=2}^{5}\left|r_{i}(k)\right|}
$$

These detection percentages depend on the physical parameters of the system (9). Table V in section IV illustrates these percentages for the taken parameters in this work (Table IV).

\section{Simulation Results}

For simulation, the following assumptions are made:

- All the robot components are in a normal mode when the robot starts to function.

- Faults are permanent.

- The challenge is to detect and isolate components wear. Hence, additive faults with small magnitudes are simulated using time varying ramp signals with different magnitudes.

Physical parameters are given in Table IV. They correspond to the Kuka KMR robot parameters. In simulations, the right diagonal forward motion is applied for $2 s$ at the beginning of simulation (the other motions give same results), with a torque vector: $\tau=[0.5,0,0,0.5]^{T}$. The angular velocity of resultant wheels 1 and 4 is $2 C_{2}=0.41 \mathrm{rad} / \mathrm{s}$.

\begin{tabular}{|c|c|c|}
\hline Parameter & Value & Unit \\
\hline$l_{x}$ & 0.1825 & $\mathrm{~m}$ \\
\hline$l_{y}$ & 0.28 & $\mathrm{~m}$ \\
\hline$R_{w}$ & 0.125 & $\mathrm{~m}$ \\
\hline$I_{z}$ & 50 & $\mathrm{~kg} \cdot \mathrm{m}^{2}$ \\
\hline$I_{w}$ & 0.89 & $\mathrm{~kg} \cdot \mathrm{m}^{2}$ \\
\hline$m$ & 390 & $\mathrm{~kg}$ \\
\hline
\end{tabular}

TABLE IV: Kuka KMR plateform physical parameters.

Using the parameters given in the Table IV, the matrix $S$ is given by:

$$
S=\left[\begin{array}{cccc}
0.6786 & 0.0904 & -0.0904 & -0.2642 \\
0.0904 & 0.6786 & -0.2642 & -0.0904 \\
-0.0904 & -0.2642 & 0.6786 & 0.0904 \\
-0.2642 & -0.0904 & 0.0904 & 0.6786
\end{array}\right]
$$

According to (21), Table IV illustrates the isolation of actuator faults using the detection percentages of each residual.

\begin{tabular}{|c|c|c|c|c|}
\hline $\begin{array}{l}\text { Detection Fault } \\
\text { percentages }\end{array}$ & $A_{1}$ & $A_{2}$ & $A_{3}$ & $A_{4}$ \\
\hline$r_{2, \text { percentage } \%}$ & 60.28 & 2.8 & 2.8 & 23.32 \\
\hline$r_{3, \text { percentage } \%} \%$ & 2.8 & 60.28 & 23.32 & 2.8 \\
\hline$r_{4, \text { percentage } \%}$ & 2.8 & 23.32 & 60.28 & 2.8 \\
\hline$r_{5, \text { percentage }}$ & 23.32 & 2.8 & 2.8 & 60.28 \\
\hline
\end{tabular}

TABLE V: Actuator fault detection percentages by the different residuals.

In what follows, some simulation results of FDI of sensor and actuator additive faults are presented to illustrate the effectiveness of the proposed method.

In Fig. 4, all residuals are presented to illustrate the signatures given in Table III. In the sequel, only significant residuals are shown. Residuals staying close to zero are not presented.

A time varying ramp signal with a slope of $10^{-2}$ is used to simulate a time varying fault on $E_{2}$. The additive signal represents an additive fault with a percentage of $10^{-2} \%$ with respect to the real value $\omega_{2}$. It is detected and isolated $0.3 \mathrm{~s}$ after its apparition as illustrated in Fig. 4.
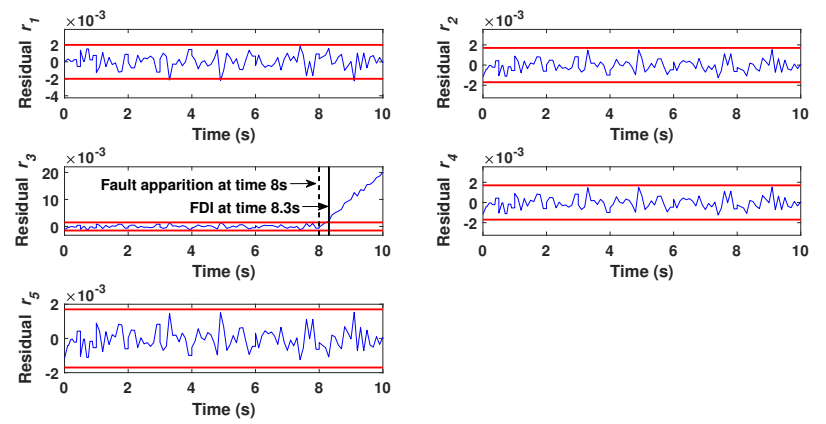

Fig. 4: $E_{2}$ fault detection and isolation.

In Fig. 5, a fault with a slope of $10^{-2}$ is simulated on the gyroscope sensor. The fault is detected and isolated just $0.22 \mathrm{~s}$ after its apparition. In Fig. 6, wheel 1 actuator $\left(A_{1}\right)$ FDI is presented. The simulated fault slope is $-10^{-2}$. The fault presents a wear on the wheel actuator. The fault is detected and isolated just $1 s$ after its apparition. 


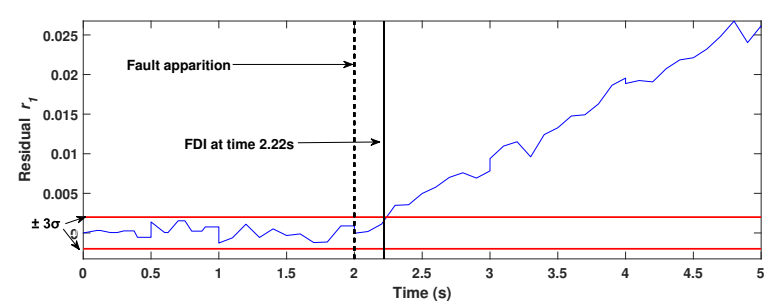

Fig. 5: Gyroscope fault detection and isolation.

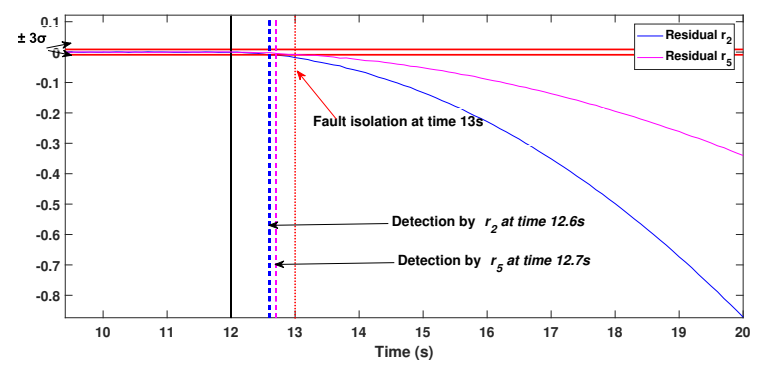

Fig. 6: $A_{1}$ fault detection and isolation.

In Fig. 7, a fault with a slope of $-10^{-3}$ is simulated in the wheel 3 actuator. It is detected and isolated $2 s$ after its apparition.

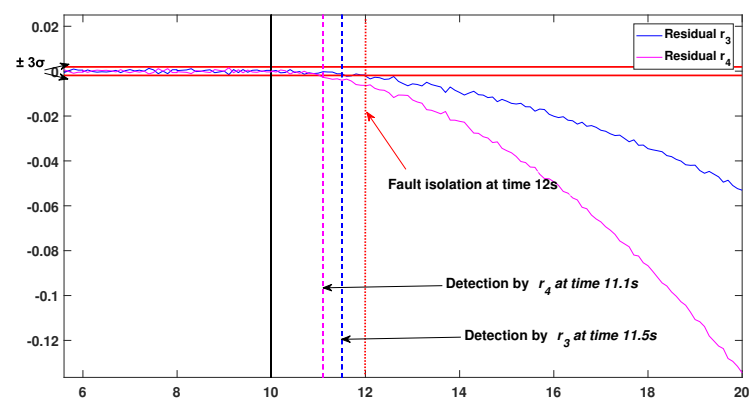

Fig. 7: $A_{3}$ fault detection and isolation.

\section{CONCLUSION}

In this paper, the problem of detecting and distinguishing wheel sensor additive faults from wheel actuator additive faults in a 4-mecanum wheeled mobile robot is studied. The considered faults have a small amplitude that they are hard to be noticed based only on the robot behaviour. These faults can represent components wear.

An approach based on an extended Kalman observer to estimate the system state and generate residuals is proposed. Sensor faults are isolated based on analyzing residual signature, while actuator faults, due to this type of robot motion properties, are more difficult to be isolated. Additional characteristics, besides on residual signatures had to be taken into consideration to isolate successfully actuator faults.

With the proposed method, such faults are detected and isolated in few seconds after their apparition. This allows to take time of planing a corrective maintenance without the need of an emergency stop of the robot. With such small faults, the robot can continue its mission without any risk of damages, until the maintenance planning.
The strongest point of the proposed approach, beside on detecting and successfully isolating wheel sensor and actuator faults in few seconds, is that it is easy to be implemented and it is very efficient.

Simulation obtained results are very promising, this is the reason for why the method is planned to be implemented sooner on a Kuka KMR omni-move plateform.

\section{REFERENCES}

[1] K. Anastassia and P. Plöger. "Model-Based Fault Diagnosis Techniques for Mobile Robots". In: IFACPapersOnLine 49 (2016).

[2] C. Zixing D. Zhuohua and Y. Jinxia. "Fault Diagnosis and Fault Tolerant Control for Wheeled Mobile Robots under Unknown Environments: A Survey”. In: Proceedings of IEEE ICRA. 2005.

[3] G.K. Fourlas, G.C. Karras, and K.J. Kyriakopoulos. "Sensors fault diagnosis in autonomous mobile robots using observer Based technique". In: ICRA. May 2015.

[4] G.K. Fourlas et al. "Model based actuator fault diagnosis for a mobile robot". In: IEEE International Conference on Industrial Technology (ICIT). Feb. 2014.

[5] O. Kost, O. Straka, and J. Dunik. "Identification of State and Measurement Noise Covariance Matrices using Nonlinear Estimation Framework". In: 659 (Nov. 2015).

[6] M. Luo et al. "Model-based fault diagnosis/prognosis for wheeled mobile robots: a review". In: 31st Annual Conference of IEEE Industrial Electronics Society, 2005.

[7] F. Pukelsheim. "The Three Sigma Rule". In: The American Statistician (1994).

[8] D. Rotondo et al. "A Fault-Hiding Approach for the Switching Quasi-LPV Fault-Tolerant Control of a Four-Wheeled Omnidirectional Mobile Robot". In: IEEE Transactions on Industrial Electronics (June 2015).

[9] H. Taheri, B. Qiao, and N. Ghaeminezhad. "Kinematic Model of a Four Mecanum Wheeled Mobile Robot". In: (Mar. 2015).

[10] C. Valdivieso and A. Cipriano. "Fault detection and isolation system design for omnidirectional soccerplaying robots". In: IEEE Conference on Computer Aided Control System Design, IEEE International Conference on Control Applications,IEEE International Symposium on Intelligent Control. 2006.

[11] P. Vlantis, C. P. Bechlioulis, and K. J. Kyriakopoulos G. Karras and G. Fourlas and. "Fault tolerant control for omni-directional mobile platforms with 4 mecanum wheels". In: IEEE ICRA. May 2016.

[12] L. Xiang and A. Zell. "Motion Control of an Omnidirectional Mobile Robot", bookTitle="Informatics in Control, Automation and Robotics: Selected Papers from the International Conference on Informatics in Control, Automation and Robotics". In: Springer Berlin Heidelberg, 2009. 